\title{
A Geometric Approach for the Modeling of Heterogeneity in Physical Structures and Processes in Water Sources.
}

\author{
Victor Peñaranda. ${ }^{1}$, Edgar Monroy. ${ }^{2}$, Iman Rousta ${ }^{3}$ \\ ${ }^{1}$ Universidad Nacional de Colombia, Colombia, vmpenarandav@unal.edu.co \\ ${ }^{2}$ Universidad Católica de Colombia, Colombia, ermonroy@ucatolica.edu.co \\ 3 Yazd University, Irán. Yazd-Irán, irousta@yazd.ac.ir
}

\begin{abstract}
Aquifers are examples of physical systems in which several phenomenologies and non-linear processes are developed, and they are highly difficult to be described. This kind of systems are depicted by attributes like the interactions between physical processes in a wide variety of scales, lithology and porous media anisotropies, heterogeneous patterns of fluid dynamics, and so on. Before this complex scenery, engineers and practitioners constantly should make decisions and to build mechanisms for understanding and use aquifers. The following manuscript shows some ideas about the analysis of complex systems and exhibiting a few fractal tools, highly useful for their applications in geophysics and groundwater systems analysis.
\end{abstract}

Keywords: heterogeneity, water sources, wetland, multifractals, complexity.

\section{INTRODUCTION}

In the face of growing population, the need to explore and take advantage of water supply sources, alternating with traditional surface water sources, is taken for granted. In Colombia, the exploitation of groundwater began several years ago by regional autonomous corporations and some private groups. They have been responsible for regulating this resource. However, it has been demonstrated in professional practice that the techniques used for the exploration, analysis and modeling of the water resources identified on underground sources are not adjusted to the complexities that participate in the dynamics of groundwater flow. For instance, the phenomena associated with the flow and transport of solutes from a wetland to aquifers are influenced by a large number of factors, among them there are heterogeneities in the porous medium and complex interactions between underground and surface water flows [1]As a result of such interactions, intrinsic structures are formed by the flow and the transport of groundwater and complex spatial patterns emerge that cannot be clearly discerned and to make decisions about their behavior and preservation is very hard [2].

In order to understand the behavior of physical properties in complex systems such as is identified in groundwater sources, new and advances techniques have evolved in the last years in the field of geophysical inspection. Moreover, new insights have been discovered into this kind of patterns; for instance, the relationship between spatial and temporal scales. These scaling properties represent the dependence of the physical (or mechanical) system to a wide range of scales on which their properties interrelate. From the first studies of the scaling properties, they have result important constant dimensionless linking the forces acting on a fluid (e.g. the Reynolds number relate viscous forces and inertial forces, or the Capillarity number relate viscous forces and surface tension in a porous medium). Another perspective of the scaling properties in geophysical patterns can be seen through the study of their geometrical properties. In this case, we are interested to understand how they are transformed changing the scales of observation. If the properties of study object are unchangeable on the scale, you may be dealing with a fractal object [3]

Fractal objects are defined as evolving symmetries, considering they are self-similar, or invariants on the scales they are observed. The physical nature of these fractal objects and how they are formed into geophysical processes are current research; however, some studies have determined that there may be a mechanism similar to that of observed in the study of phase transitions [4] [5]. Applications of phase transitions into our context provide a group of innovative tools for: (i) explaining how preferential flow is formed on a porous medium, ii) understanding how interactions are 
emerged between the scales of the observed physical process, and iii) providing information on the space organization - temporal fractal structures found in the general theory of groundwater flow.

Based on our literature inspection, there is a limited knowledge about the groundwater flow properties and applications for modeling sceneries with high precisions are still a scientific challenge. In current deterministic models, hydraulics properties of the porous media (i.e. conductivity, porosity, water content) are characterized and they are considered to be homogeneous in the whole space. In stochastic models, heterogeneities are better described, but some statistical assumptions are necessary to be considered (i.e. stationarity, ergodicity or normality). Another kind of models, based on fractal theory, are more efficient and effective to explain heterogeneities and relations among scales. These models have a less number of parameters in their mathematical structures and they preserved the geometrical properties of observed patterns [6] [7] [8]. In this paper, we are going to show some insight about two models based on fractal theory and how they can be approach for understanding heterogeneities and physical properties in aquifers.

\section{METHODS.}

\section{a. Fractal - Multifractal Approach}

Concepts of fractals and multifractals were formally introduced by Mandelbrot [9] in order to explain the behavior of complex geometric shapes. Fractals are defined as geometric patterns repeating themselves over a wide range of scales (self-similarity) and their scaling properties are represented by power laws. Moreover, multifractals are also geometric objects with self-similar properties but those properties cannot be described by a unique power law [10]. Either fractals or multifractals are used for the description of physical systems heterogeneity or they have been very useful in a wide number of applications related to the study of porous media [6] [11] [12] [13]

Among the models that use concepts from the fractal and multifractal theory, the Fractal - Multifractal (FM) approach is highlight here. This model was introduced by Puente, (1996), [10]. For the deterministic description of rainfall fields but its applications have been extended to the study of solute transport processes in porous media [11]. In the FM approach, observations are constructed through projections from a multifractal measure which lies in the geometrical space of a Fractal Interpolation Function (FIF). These functions were initially worked by Barnsley [2] as an application of Iterated Function Systems (IFS). However, [10] took Barnsley's ideas exploring the behavior of the statistical distribution of points that form the IFS. As a result, a new approach for geophysical patterns was evolved.

During the construction of FIF in the $\mathbb{R}^{2}$ plane, two statistical measure are generated when the relative frequencies of the $\mathrm{x}$ and $\mathrm{y}$ axes are counted. Given the continuity of the FIF, the $\mathrm{y}$-axis frequencies are interpreted as a generated measure from the $x$-axis frequencies through the transformation equation $f_{z}(x)=y$. This interpretation is alike to the concept of generated probability distributions, commonly used for engineering applications (e.g. for getting random numbers). Depending on how the FIF points are distributed in the $\mathbb{R}^{2}$ (Equation 1) plane and the parameters are stated, these generated measured can possess multifractals properties highly well defined.

In order to get a unidimensional generated measure with the FM model, the FIF should be defined for the $\mathrm{N}+1$ points in the $\mathbb{R}^{2}$ (Equation 2) plane and for all $\mathrm{N}$ affine contractile maps of the form:

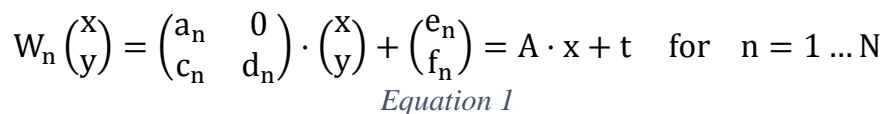

Under the following restrictions:

$$
\mathrm{W}_{\mathrm{n}}\left(\begin{array}{l}
\mathrm{x}_{0} \\
\mathrm{y}_{0}
\end{array}\right)=\left(\begin{array}{l}
\mathrm{x}_{\mathrm{n}-1} \\
\mathrm{y}_{\mathrm{n}-1}
\end{array}\right), \quad \mathrm{W}_{\mathrm{n}}\left(\begin{array}{l}
\mathrm{x}_{\mathrm{N}} \\
\mathrm{y}_{\mathrm{N}}
\end{array}\right)=\left(\begin{array}{l}
\mathrm{x}_{\mathrm{n}} \\
\mathrm{y}_{\mathrm{n}}
\end{array}\right) \quad 0 \leq \mathrm{d}_{\mathrm{n}} \leq 1
$$


In this model, $\left\{a_{n}, c_{n}, d_{n}, e_{n}, f_{n}\right\}$ are the FM-approach parameters which can be derived by the model restrictions. However, the parameter $d_{n}$ is bounded on $[0,1]$ and defined by the modeler. When all the model conditions are satisfied, there exists a unique "fix point" representing by the G-plot (Equation 3) of the FIF f: $x \rightarrow y$, such that $G=$ $\{(\mathrm{x}, \mathrm{f}(\mathrm{x})) \mid \mathrm{x} \in \mathbb{R}, \mathrm{y} \in \mathbb{R}\}$ is obtained by an iterative procedure defined under the construction of affine contractile maps:

$$
\mathrm{G}=\bigcup_{\substack{\mathrm{n}=1 \\ \text { Equation } 3}}^{\mathrm{N}} \mathrm{W}_{\mathrm{n}}(\mathrm{G})
$$

If a rotation $\theta$ is applied to the points defined by the affine contractile maps, a new parameter evolves in the model and also new complex patterns emerge. The definitions of projections of (multifractal) generated measures for different values of the angle $\theta$ (Equation 4), can be understood as an affine transformation in the Euclidean plane $\mathrm{W}: \mathbb{R}^{2} \rightarrow \mathbb{R}^{2}$, where $\mathrm{W}_{\mathrm{n}}(\mathrm{x})$ is defined by:

and

$$
W_{n}(x)=A_{n} \cdot x+t_{n}
$$

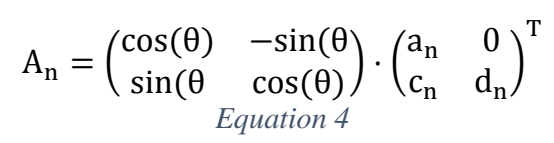

is a matrix which changes the relative space through rotation and contractions. Similarly, the FM approach can be extended to the $\mathbb{R}^{3}$ (Equation 5) plane if the FIF is defined for the $N+1$ points onf $\mathbb{R}^{3}$ (Equation 6) and for the $N$ contractile maps of the form:

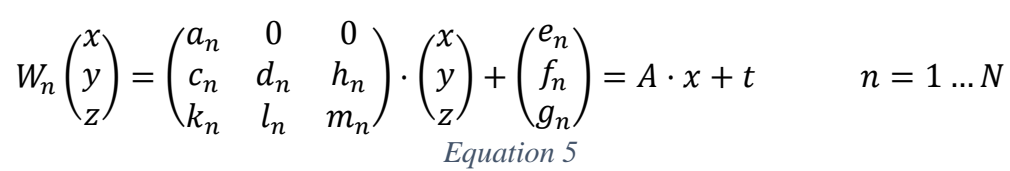

Under the restrictions:

And the spectral norm of,

$$
W_{n}\left(\begin{array}{l}
x_{0} \\
y_{0} \\
z_{0}
\end{array}\right)=\left(\begin{array}{l}
x_{n-1} \\
y_{n-1} \\
z_{n-1}
\end{array}\right), \quad W_{n}\left(\begin{array}{l}
x_{N} \\
y_{N} \\
z_{N}
\end{array}\right)=\left(\begin{array}{l}
x_{n} \\
y_{n} \\
z_{n}
\end{array}\right)
$$

$$
\begin{gathered}
\psi=\left(\begin{array}{ll}
d_{n} & h_{n} \\
l_{n} & m_{n}
\end{array}\right) \\
\text { Equation } 6
\end{gathered}
$$

should be less than one, i.e. root square of the maximum value in the product of $\psi$ (Equation 7) and its transpose $\psi^{T}$ should be less than one:

$$
\|\psi\|_{2}=\underset{\text { Equation } 7}{\sqrt{\lambda_{\max }\left(\psi \psi^{T}\right)}}<1
$$

If the aforementions restrictions are fullfiled, a unique plot $G$ of the FIF results and $G=\left\{\left(x, y, f_{z}(x, y)\right) \mid x \in \mathbb{R}, y \in\right.$ $\mathbb{R}, z \in \mathbb{R}\}$ (Equation 8) should satisfy

$$
G=\bigcup_{\substack{n=1 \\ \text { Equation } 8}}^{N} W_{n}(G)
$$


The FIF is built with 4 free parameters $\left\{d_{n}, h_{n}, l_{n} m_{n}\right\}$ and $N+1$ points. Nonetheless, the generated measure can be also transforms by a rotation parameter $\theta$ (Equation 9 ), which is applied the free parameters, such that

$$
A_{n}=\left(\begin{array}{cc}
\cos (\theta) & -\sin (\theta \\
\sin (\theta & \cos (\theta)
\end{array}\right) \cdot\left(\begin{array}{cc}
d_{n} & h_{n} \\
l_{n} & m_{n}
\end{array}\right)^{T}
$$

The FM-approach can now be applied in groundwater hydrology to describe highly heterogeneous and intermittent observed patterns. In figure 1 is illustrated a generated solute concentration field which exhibits heterogeneity of solutes through the porous media (see tridimensional frame). Some other projections of the tridimensional patterns are also exhibit in figure 1 . One of them is a multifractal pattern (see the $f(x)$ projection).

To identify the connection between the FM-approach parameters and the physics of porous media is still a research problem, however getting a parsimonious (i.e. less parameters) and geometric representation of patterns is already an advantage. The kind of geometry that exhibits the FM-approach shows a non-linear dynamics of groundwater processes and their representation can optimistically replace the complex non-linear differential equations that are used initially to explain their physics. The new stages of the FM-approach should be guided to face more complex problems; for instance, getting a space-time framework for describing evolving patterns and of course, the physics of parameters.

\section{RESULTS.}

\section{a. Multiplicative Random Cascades}

Many of the multifractal models are mathematical constructions that seek to capture the intermittence and irregular behavior of natural observables. One of them are the multiplicative random cascades which were initially applied to the study of turbulence [14] [15] [16]. These ideas about the description of complex patterns based on multiplicative processes allows to be extended to others field of physics. The concept of multiplicative processes is also based on the idea of self-similarity, i.e. physical quantities that are composed of themselves. In the construction of multiplicative random cascades, the generated measures $M_{n}(x)$ (Equation 10) with density $\rho_{n}(x)$ are defined by

$$
\rho_{n}(x)=\prod_{k=1}^{n} W_{\left\langle j_{1}, \ldots, j_{k}\right\rangle} x \in \Delta_{n}\left(j_{1}, \ldots, j_{k}\right)
$$

where $W_{\left\langle j_{1}, \ldots, j_{k}\right\rangle}$ are positive iid r.v. (independent and identically distributed random variable). Being $W_{\left\langle j_{1}, \ldots, j_{k}\right\rangle}$ a random variable, the name of multiplicative random cascade is now well defined. 

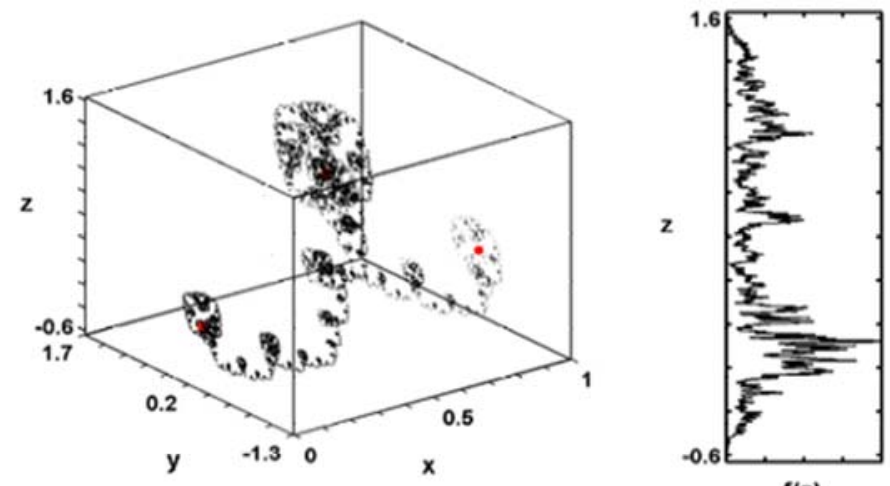

$f(z)$
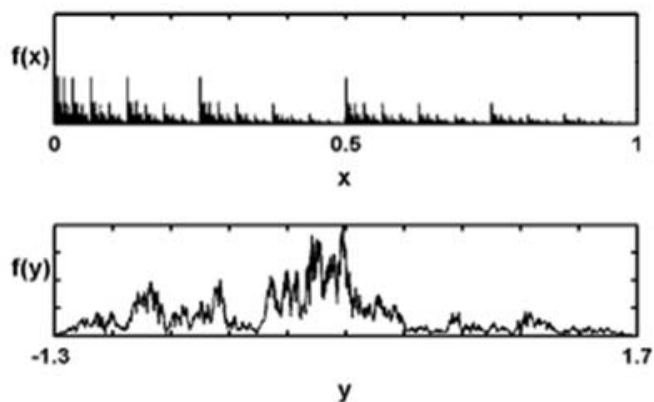

Fig. 1. The tridimensional frame shows a solute concentration field in a porous media which is generated by the parameters $\theta=90^{\circ}, d_{n}=0.5, h_{n}=0.0, l_{n}=0.0, m_{n}=0.5$ and FIF points: $p_{1}=\{0,0,0\}, p_{2}=\{0.5,0.2,1\}, p_{3}=$ $\{1,0,0\}$. To the left and below the tridimensional frame are exhibited generated measured $f_{z}(x)=f(x), f_{z}(y)=f(y)$ y $f_{x, y}(z)=f(z)$ (Equation 11), and they result during the building process of the FIF.

$$
b=\prod_{\substack{i=1 \\ \text { Equation } 11}}^{d} b_{i} \quad d<1
$$

Where $b_{i}$ represents the number of subdivisions in every step of the cascade constructions. The sub-cubes under $n$ subdivisions are denoted by $\Delta_{n}^{i}$ and its length by $L_{n}$. Initially, the $d$-dimensional $\left[0, L_{0}\right]^{d}$ cube have a mass $M_{0} L_{0}^{d}$ and to the first sub-divisions of cubes are assigned a mass $\mu_{1}\left(\Delta_{1}^{i}\right)=M_{0} L_{0}^{d} W_{1}^{i} / b$ for $i=\{1,2, \ldots, b\}$. In this mathematical expression, $W_{n}^{i}$ represents the generator of iid r.v. For every subdivision $\Delta_{n}^{i}$ of the cascade, a mass $\mu_{n}\left(\Delta_{n}^{i}\right)$ is associated and it is defined by the product between the starting mass $M_{0} L_{0}^{d}$ and the mathematical product of every $W_{n}^{i}$ related to the sub-subes $\Delta_{n}^{i}$ (Equation 12), such that:

$$
\mu_{n}\left(\Delta_{n}^{i}\right)=\frac{M_{0} L_{0}^{d}}{b^{n}} \prod_{j-1}^{n} W_{j}^{i}
$$

Equation 12

The limit mass $\mu_{\infty}\left(\Delta_{n}^{i}\right)$ is obtained when $n \rightarrow \infty$ (Equation 13), satisfying the recursive equation:

$$
\mu_{\infty}\left(\Delta_{n}^{i}\right)=\mu_{n}\left(\Delta_{n}^{i}\right) \frac{\mu_{\infty}\left(\left[0, L_{0}\right]^{d}\right)}{R_{0} L_{0}^{d}}=\mu_{n}\left(\Delta_{n}^{i}\right) Z_{\infty}(i) \quad i=\left\{1,2, \ldots, b^{n}\right\}
$$


Where $Z_{\infty}(i)$ is statistically independent of $\mu_{n}\left(\Delta_{n}^{i}\right)$ and represents the high-frequency component in the structure of the random cascade (small scale elements). On the other hand, $\mu_{n}\left(\Delta_{n}^{i}\right)$ represents the low-frequency elements in the random cascade and joint to $Z_{\infty}(i)$, highly intermittent patterns are built. In Over and Gupta's model, the mass is conserved during the cascade construction and for getting such a conservation is necessary that the expected value of the random generator equals one, i.e. . $E[W]=1$ and $E\left[Z_{\infty}\right]=1$.

The theory of random cascades can be also applied in the study of transport processes in aquifers, in the same way as it was illustrated for the FM approach. In figure 2 is illustrated an application case of multiplicative random cascade with Over and Gupta's model. This cascade is simulated for the parameters $d=2$ and $b=4$. Based on the results of the model high spatial intermittency of the solute concentration field is also obtained, likewise, high heterogeneity of the physical medium is also achieved.

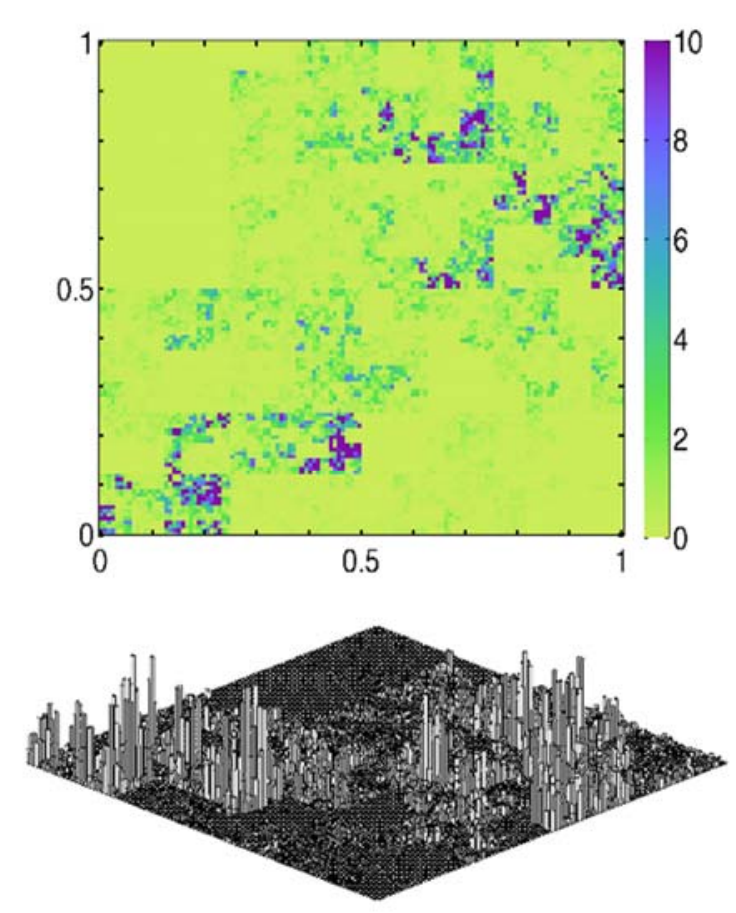

Fig 2. Ilustration of a multipicative random cascade simulation with the Over and Gupta's model for getting a solute concentration field in a porous media. This simulation was generated for a re-scale range $z=[0,10]$ and the used parameters were $d=2, b=4, M_{0} L_{0}^{2}=1$ and $E[W]=1$. The upper frame shows a plan view of the tridimensional object illustrated in the lower frame. Both frames are illustrating the intermittency and heterogeneity of a solute concentration field.

\section{IV.CONCLUSIONS.}

Multifractal formalism for aquifer systems modelling have been widely applied by scientists and practitioners for characterizing groundwater observations. From the results of various studies in this research field, the progress has been outstanding for understanding nonlinear physical processes with high heterogeneity as the porous media fields exhibit. However, the progress of the models is still under construction and several weaknesses should be solved for getting a better insight of how groundwater processes work. As an example of the challenges is to understand the transport of porous media and its relationship on different observations scales. 
Some of the conceptual models that have been introduced here for understanding heterogeneity in aquifers and this intro have been shown in order to generate concerns in readers and to anticipate questions about the usefulness of them to face problems in groundwater engineering. Nevertheless, it is important to have on consideration that the simplicity of these models and their connection with the geometric properties of patterns are attractive and innovative. Perhaps through a clear understanding of the geometric and symmetry breaking properties of the physical system, we will be able to improve our capability for prediction.

\section{REFERENCES}

[1] Sivakumar, B., Harter, T. y Zhang, H. (2005). Solute transport in a heterogeneous aquifer: A search for nonlinear deterministic dynamics. Nonlinear Processes in Geophysics, 12, 211-218

[2] Barnsley, M. (1993). Fractals Everywhere (Segunda Ed.). Morgan Kaufmann Pub.

[3] Feder, J. (1988). Fractals. New York and London: Plenum Press.

[4] Hunt, A. G, 2001, Applications of percolation theory to porous media with distributed local conductances, Advances in Water Resources, 24(3,4): 279-307

[5] Hunt, A. y Ewing, R. (2017). Scaling. In Cushman, J. y Tartakovsky, D. (Eds.). The handbook of groundwater engineering (3rd edition) (pp. 459-495). Boca Raton, Florida, USA: The Taylor \& Francis Group.

[6] Folorunso, O., Puente, C., Rolson, D. y Pinzon, J. (1994). Statistical and fractal evaluation of the spatial characteristics of soil surface strength. Soil Science Society of America Journal, 58, 284-294.

[7] Molz, F., Liu, H. y Szulga, J. (1997). Fractional Brownian motion and fractional Gaussian noise in subsurface hydrology: a review, presentation of fundamental properties, and extensions. Water Resources Research, 33(10), 2273-2286.

[8] Molz, F., H. Rajaram, y S. Lu. (2004). Stochastic fractal-based models of heterogeneity in subsurface hydrology: origins, applications, limitations, and future research questions. Reviews of Geophysics, 42, RG1002, doi:10.1029/2003RG000126.

[9] Mandelbrot, B. (1982). The fractal geometry of nature. New York: W.H. Freeman.

[10] Neuman, S. (1990). Universal scaling of hydraulic conductivities and dispersivities in geologic media, Water Resources Research, 26, 17491758.

[11] Pfeifer, P., Avnir, D., y Farin, D. (1983). Ideally irregular surfaces of dimension greater than two in theory and practice. Surface Science, 126, 569-572.

[12] Puente, C. (1996). A new approach to hydrologic modeling: derived distributions revisited. Journal of Hydrology, 187 (1,2), 65-80.

[13] Puente, C., Robayo, O., Diaz, M. Y Sivakumar, B. (2001). A fractal-multifractal approach to groundwater contamination. 1. modeling conservative tracers at the borden site. Stochastic Environmental Research and Risk Assessment, 15(5), 357-371

[14] Frisch, U. (1995). Turbulence: The legacy of A. N. Kolmogorov. Cambridge University Press.

[15] Frisch, U. y Parisi, G. (1985). On the singularity structure of fully developed turbulence.

[16] Vargas, ERM, \& Gonzalez, JP (2013). conceptual hydrogeologic model and analysis of vulnerability to contamination of the aquifer Duitama Boyacá-Colombia. In Vestigium Ire, 3 (1).

\section{AUTHOR PROFILE}

Victor Peñaranda. Professor of engineering research, engineering faculty, Juan de Castellanos University Edgar Monroy. Professor of engineering research, engineering faculty, Catholic University of Colombia Iman Rousta. Assistant professor in Climatalogy, Faculty of Humanities, Department of Geography, Yazd University 